\title{
Strontium ranelate treatment in a postmenopausal woman with osteonecrosis of the jaw after long-term oral bisphosphonate administration:
}

\section{a case report}

\author{
This article was published in the following Dove Press journal: \\ Clinical Interventions in Aging \\ II July 2017 \\ Number of times this article has been viewed
}

\author{
Whei-Lin Pan ${ }^{1,2}$ \\ Pi-Lun Chen ${ }^{2}$ \\ Cho-Ying $\operatorname{Lin}^{1,2}$ \\ Yi-Chun Pan ${ }^{2}$ \\ Yuh-Ren Ju',2 \\ Chiu-Po Chan ${ }^{1,2}$ \\ Robert WW Hsu ${ }^{2,3}$ \\ 'Department of Periodontics, \\ Chang Gung Memorial Hospital, \\ Taipei, Taiwan; ${ }^{2} \mathrm{Graduate}$ Institute \\ of Dental and Craniofacial Science, \\ Chang Gung University, Taoyuan, \\ Taiwan; ${ }^{3}$ Department of Orthopedics, \\ Chang Gung Memorial Hospital, \\ Taipei, Taiwan
}

\begin{abstract}
Bisphosphonates (BPs) suppress bone resorption and increase bone strength, thus reducing the risk of fracture. Oral BPs are widely used for the prevention and treatment of osteoporosis and osteopenia. Here, we describe the case of a postmenopausal woman who took oral alendronate for $>3$ years for osteoporosis. The patient presented at the clinic with sharp jaw pain and swelling on the left mandible 4 months after extraction of the third molar. Clinical examinations identified an inflamed mucosal opening with pus over an area of necrotic bone. Initial images of cone beam computed tomography revealed a sequestrum at the extracted socket. The condition did not improve after 1 week of antibiotic treatment; therefore, the alendronate treatment was terminated and the patient was prescribed strontium ranelate instead. The patient gradually recovered and, at the 2-year follow-up, the site of BP-related osteonecrosis of the jaw healed completely as determined by both clinical and cone beam computed tomography measures. The bone mineral densities in the femoral neck and lumbar spine improved after 1 year, and were maintained at the 3-year follow-up. The serum C-terminal cross-linking telopeptide values also gradually increased from the initial $130 \mathrm{pg} / \mathrm{mL}$ to $320 \mathrm{pg} / \mathrm{mL}$ at the 3 -year follow-up. Taken together, this case supports the use of strontium ranelate as an alternative treatment for postmenopausal women who receive long-term oral BP treatments and are at risk for serious complications of BP-related osteonecrosis of the jaw.
\end{abstract}

Keywords: bisphosphonate-related osteonecrosis of the jaw, BRONJ, bisphosphonates, strontium ranelate

\section{Introduction}

Bisphosphonate-related osteonecrosis of the jaw (BRONJ) is a rare but severe complication. ${ }^{1}$ The disease is characterized by the exposed bone for over 8 weeks in the maxillofacial region after antiresorptive or antiangiogenic therapy, with no history of radiation therapy or metastatic disease to the jaws. ${ }^{2}$ Intravenous bisphosphonates (BPs) are mostly used to manage cancer-related bone disease, while oral BPs are common first-line medications for treating osteoporosis. Although the incidence of BRONJ is higher in patients receiving intravenous BPs, more patients suffer from oral BRONJ, considering the global population taking oral BPs. ${ }^{3}$ In fact, the risk of osteoporosis patients to develop ONJ is associated with the use of the oral BP alendronate. ${ }^{3}$ Therefore, alternative treatments are needed for osteoporosis patients who have developed BRONJ or are at high risk for the disease.
Correspondence: Whei-Lin Pan Chang Gung Memorial Hospital, 199 Tung Hwa North Road, 105 Taipei, Taiwan

Tel +886227602407

Fax +88622756 2221

Email helen481209@gmail.com 
Strontium ranelate (SR) is a unique anti-osteoporosis medication, which is suggested to have dual effects on bone metabolism. ${ }^{4}$ In vitro studies show that SR acts through the calcium-sensing receptor to increase the mRNA level of osteoprotegerin and decrease that of receptor activator of nuclear factor-kappaB ligand. ${ }^{4} \mathrm{SR}$ is shown to promote bone formation by stimulating the differentiation of osteoblasts and to block bone resorption by inhibiting osteoclast differentiation. ${ }^{5}$ Also, a 10-year clinical trial finds that SR reduces the risk of vertebral and non-vertebral fractures, and increases bone mineral density (BMD). ${ }^{6}$ Yet, few reports detail surgical interventions followed by daily SR for osteoporosis patients suffering BRONJ. ${ }^{7}$ Here, we present the case of a postmenopausal woman with osteoporosis who suffered from ONJ related to long-term oral BP consumption. We describe the treatment of BRONJ and report the findings from 3 years of follow-up visits after switching from BP therapy to SR treatment.

\section{Case report}

An 83-year-old woman presented at the clinic with persistent deep ache in the left posterior mandible following the extraction of her third molar 4 months ago. The patient experienced severe gingival swelling and pain that interfered with the use of denture. A review of the medical record showed that the patient had a history of osteoporosis and had been taking once-weekly alendronate/vitamin D3 70 mg/2,800 IU (Fosamax ${ }^{\circledR}$ Plus D, Merck, Kenilworth, NJ, USA) for $>3$ years. The patient reported no other pre-existing medical conditions such as cardiovascular disease, diabetes mellitus, blood dyscrasia, etc., and had not taken any steroids or other medications known to affect bone metabolism within the past 5 years.

Clinical examination identified an $8 \times 6 \mathrm{~mm}$ gingival swelling with pus draining through gingival fistulas (Figure 1A). The surrounding mucosa appeared red, edematous, and bled on probing. Initial periapical radiographs
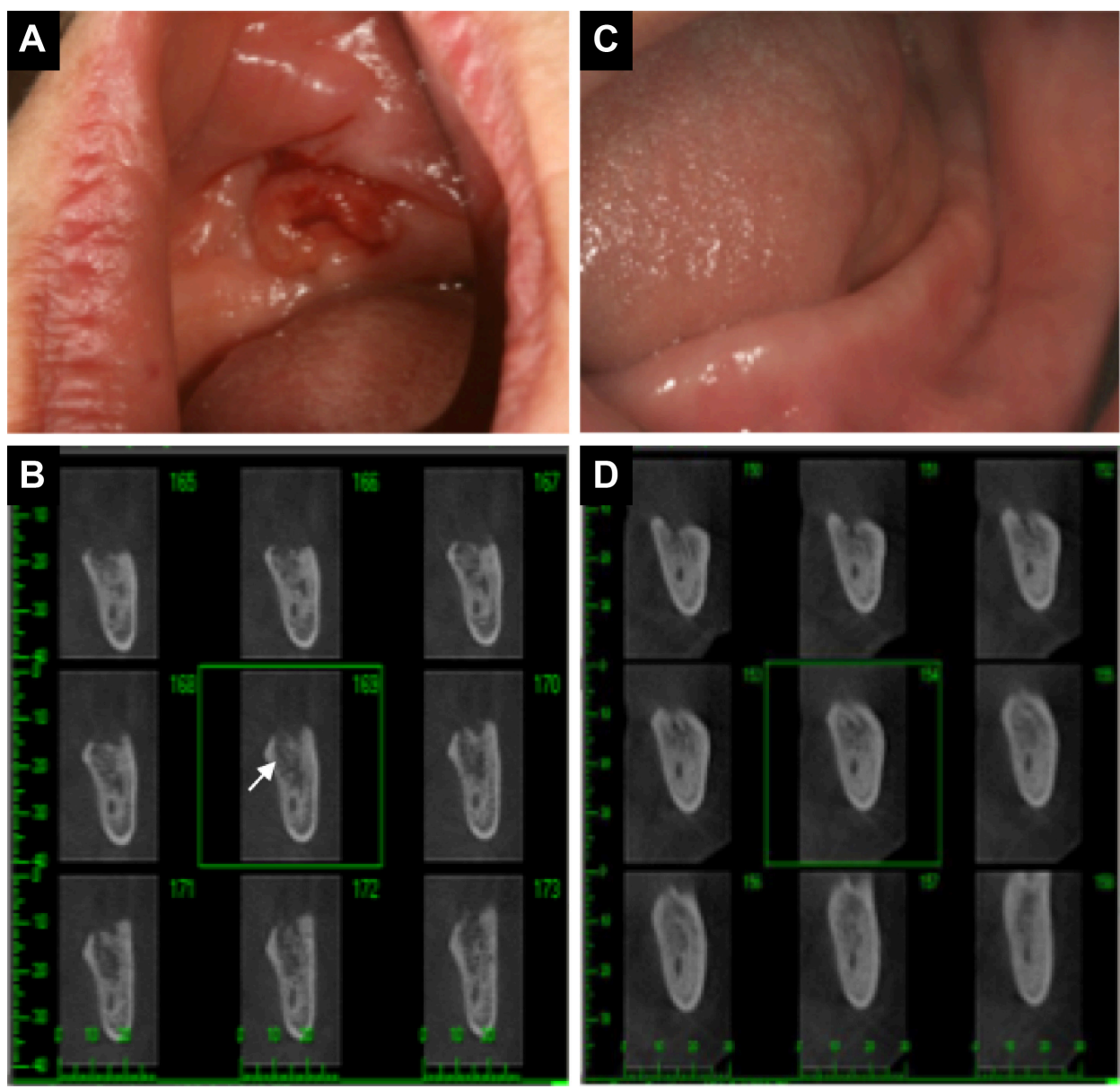

Figure I Images of the areas affected by BRONJ.

Notes: (A) The initial appearance surrounding the extraction socket of lower left third molar, showing red and swollen gingiva with pus draining through gingival fistulas. (B) Initial cross-sectional CBCT images showed rarefaction of trabecular bone with a large area of osteonecrotic lesion around the extraction socket (as indicated by the white arrow). (C) After 2 years of SR treatment, the gingiva over the extraction socket is healthy with no sign of gingival inflammation. (D) After 2 years of SR treatment, $\mathrm{CBCT}$ images demonstrated that the bone density increased and new spongy bone had begun to fill into the area previously occupied by dead bone.

Abbreviations: BRONJ, bisphosphonate-related osteonecrosis of the jaw; CBCT, cone beam computed tomography; SR, strontium ranelate. 
showed a radiolucent shadow, suggesting osteolysis in this area. The cone beam computed tomography (CBCT) confirmed apparent osteolysis and osteosclerosis around the left edentulous area corresponding to the extraction socket (Figure 1B). The fasting serum C-terminal cross-linking telopeptide (CTX) was tested to evaluate the level of bone resorption. ${ }^{8}$ The initial CTX value was $130 \mathrm{pg} / \mathrm{mL}$ (Table 1), indicating little bone turnover. The patient was prescribed penicillin $500 \mathrm{mg}$ by mouth 4 times a day, analgesics for pain management, and $0.12 \%$ chlorhexidine oral rinse twice a day. The wound showed significant improvement within 1 week of antibiotic coverage. Unfortunately, the patient returned 3 weeks later and was again suffering from pain and gingival swelling with fistulas in the same area. After consulting with the primary care physician and orthopedic surgeon, alendronate was discontinued and the patient was switched to $2 \mathrm{~g}$ of SR (Protos, Servier, Suresnes, France) daily. Mouth rinse was still prescribed to control oral infection.

Two months after the switch from BP to SR, the serum CTX value of the patient increased to $160 \mathrm{pg} / \mathrm{mL}$, and we performed a conservative debridement and removed the sequestrum on the superficial layer of alveolar bone with a curette to obtain primary closure. At the 6- and 12-month follow-up visits, the serum CTX measurements were $220 \mathrm{pg} / \mathrm{mL}$ and $280 \mathrm{pg} / \mathrm{mL}$, respectively, suggesting recovery in bone turnover (Table 1). At the 24-month follow-up, the affected site had healed completely, as determined by clinical and radiographical measures. The gingiva surrounding the extraction socket displayed no sign of inflammation or fistula (Figure 1C). The CBCT images showed increased bone density with no sign of osteolysis, and newly formed spongy bone was detected occupying the sites of previously necrotic area (Figure 1D). BMD increased in the femoral neck (T-score from -1.3 to -0.6 ) and in the lumbar spine (T-score from -2.5 to -0.4 ) after 1 year of treatment with SR. Three years after the switch from BP to SR, the T-score showed little to no change when compared with the value at 1-year follow-up (Table 1), indicating that the BMD was sustained. The serum CTX value reached $320 \mathrm{pg} / \mathrm{mL}$, suggesting a

Table I Tests for osteoporosis risk assessment

\begin{tabular}{llll}
\hline & $\begin{array}{l}\text { Initial-before the } \\
\text { discontinuation } \\
\text { of BPs }\end{array}$ & $\begin{array}{l}\text { I year after } \\
\text { switching } \\
\text { from BP to SR }\end{array}$ & $\begin{array}{l}\text { 3 years after } \\
\text { switching } \\
\text { from BP to SR }\end{array}$ \\
\hline BMD T scores & & \\
In femoral neck & $-\mathrm{I} .3$ & -0.6 & -0.5 \\
In lumbar spine & -2.6 & -0.4 & -0.4 \\
CTX (pg/mL) & I 30 & 280 & 320
\end{tabular}

Abbreviations: BMD, bone mineral density; BPs, bisphosphonates; CTX, C-terminal cross-linking telopeptide; SR, strontium ranelate. healthy rate of bone remodeling. Therefore, combined treatments of initial oral antibiotics and sequestrectomy, together with switching from BP to SR, allowed the patient to recover from BRONJ. In this case, SR treatment effectively increased and maintained the BMD of the patient.

\section{Discussion}

BPs are stable analogs of naturally occurring inorganic pyrophosphate, which is a by-product of cellular metabolism. ${ }^{3}$ BPs bind to hydroxyapatite crystals of the bone with a high affinity and inhibit hydroxyapatite breakdown. By associating with the bone, BPs are preferentially taken up by osteoclasts and can inhibit bone resorption by promoting the apoptosis of osteoclasts. ${ }^{9}$ The alveolar bone, especially in the mandible, shows the fastest remodeling rate in the adult skeleton, resulting in high levels of BP accumulation. ${ }^{10}$ However, since bone resorption is an important aspect of normal bone metabolism, high levels of BPs may inhibit bone remodeling to the point that microdamages cannot be repaired, leading to the development of ONJ. ${ }^{11}$

The incidence of ONJ associated with oral BP consumption has not been well documented. A recent systematic review showed that the incidence of BRONJ is estimated to be $0.47 \%$ for oral BP treatments, compared with $6.9 \%$ for intravenous BPs administration. ${ }^{12} \mathrm{Up}$ to $80 \%$ of all BRONJ cases were associated with invasive dental procedures involving bone exposure, that is, tooth extraction, root-tip resections, periodontal surgery, etc. ${ }^{12}$ Other major risk factors include age, gender, the type/dosage/duration of BP exposed, comorbidities such as rheumatoid arthritis or diabetes, and concomitant administration of medications such as corticosteroids. ${ }^{2}$ In the case described here, BRONJ occurred as an adverse event of a $>3$-year oral BP therapy.

While oral BPs are the most frequently prescribed medications for osteoporosis treatment, patients who have unsatisfactory responses or develop BP-related adverse effects (such as BRONJ or esophagitis) are advised to use other medications. Among other common medications, teriparatide is the only one that has been shown to stimulate bone formation and may reverse osteoporosis to some extent. ${ }^{13}$ However, teriparatide is recommended only for patients who are at high risk for fracture and cannot tolerate other medications, because of the cost and lack of long-term safety data. ${ }^{13} \mathrm{SR}$ is also commonly prescribed by clinicians and has been suggested to show dual effects of promoting bone formation and inhibiting bone resorption in both in vitro and in vivo studies. ${ }^{5,14}$ In addition, SR was shown to have beneficial effects on bone microarchitecture in both cortical and trabecular bone. ${ }^{15}$ Lima et al followed the effects of SR 
in a group of postmenopausal women with osteoporosis who were previously treated with oral BPs. ${ }^{16}$ They showed that SR has short-term stimulating effect on bone formation, and is associated with a long-term increase in BMD. Longato et al reported a case of BRONJ in a patient with rheumatoid arthritis. $^{7}$ The patient was switched from BP to SR after the occurrence of BRONJ, but the effect of SR treatment was not reported. In this report, the outcome showed clear anabolic activity after discontinuing alendronate and the use of SR, as monitored by clinical, radiographical and biochemical examinations for 3 years. It is worth noting that BP has been reported to have a skeletal half-life as long as 8-15 years. ${ }^{17-19}$ It was not clear if the patient was still under the influence of alendronate after being switched to SR, but some experts agree that the discontinuation of BP treatment promotes wound healing in BRONJ patients. ${ }^{3,20,21}$

Clinical treatment of BRONJ consists of conservative therapy, including pain relief, and the use of topical and systemic antibiotics to limit secondary infection and prevent the extension of the exposed bone. Localized, conservative sequestrectomy and surgical debridement is recommended only for treating advanced, nonresponsive disease. Fasting serum CTX values of $<100 \mathrm{pg} / \mathrm{mL}$ has been suggested in dentistry as a high risk of developing BRONJ after oral surgeries. ${ }^{22}$ However, the reliability of this assessment remains controversial. Some studies confirm the association between low CTX values and the development of BRONJ after surgery, ${ }^{23,24}$ but other studies report the lack of correlations between CTX values with either surgery-related BRONJ or oral/systematic BMD. ${ }^{25,26}$ In this case, we showed that the CTX values continued increasing up to 3 years of SR treatment. Meanwhile, BMD increased within the first year of SR treatment, and was maintained at the 3-year follow-up. These data support a healthy level of bone turnover and well-maintained BMD under SR treatment.

In conclusion, we described a postmenopausal woman who developed oral BRONJ after the extraction of the lower left third molar. The BRONJ symptoms were successfully treated with antibiotics, conservative surgical debridement, and chlorhexidine mouth rinse. The patient was switched from oral alendronate to strontium ranelate, and demonstrated satisfactory recovery of bone turnover and BMD in clinical, radiographic, and biochemical examinations over the 3-year follow-up period.

\section{Acknowledgment}

Written informed consent was obtained from the patient for publication of this case report and any accompanying images.

\section{Disclosure}

The authors report no conflicts of interest in this work.

\section{References}

1. Marx RE, Stern D. Oral and Maxillofacial Pathology. Chicago, IL: Quintessence; 2003:36-38.

2. Ruggiero SL, Dodson TB, Fantasia J, et al. American Association of oral and maxillofacial surgeons position paper on medication-related osteonecrosis of the jaw 2014 update. J Oral Maxillofac Surg. 2014; 72(10): 1938-1956.

3. Paiva-Fonseca F, Santos-Silva AR, Della-Coletta R, Vargas PA, Lopes MA. Alendronate-associated osteonecrosis of the jaws: a review of the main topics. Med Oral Patol Oral Cir Bucal. 2014;19(2): e106-e111.

4. Bonnelye E, Chabadel A, Saltel F, Jurdic P. Dual effect of strontium ranelate: stimulation of osteoblast differentiation and inhibition of osteoclast formation and resorption in vitro. Bone. 2008;42(1):129-138.

5. Trouvin AP, Goeb V. Receptor activator of nuclear factor-kappaB ligand and osteoprotegerin: maintaining the balance to prevent bone loss. Clin Interv Aging. 2010;5:345-354.

6. Reginster JY, Kaufman JM, Goemaere S, et al. Maintenance of antifracture efficacy over 10 years with strontium ranelate in postmenopausal osteoporosis. Osteoporos Int. 2012;23(3):1115-1122.

7. Longato L, Cavalli L, Marcucci G, et al. Osteonecrosis of the jaw in a patient with rheumatoid artritis treated with an oral aminobisphosphonate: a clinical case report. Clin Cases Miner Bone Metab. 2013;10(2): 139-141.

8. Hutcheson A, Cheng A, Kunchar R, Stein B, Sambrook P, Goss A. A C-terminal crosslinking telopeptide test-based protocol for patients on oral bisphosphonates requiring extraction: a prospective single-center controlled study. J Oral Maxillofac Surg. 2014;72(8):1456-1462.

9. Plotkin LI, Manolagas SC, Bellido T. Dissociation of the pro-apoptotic effects of bisphosphonates on osteoclasts from their anti-apoptotic effects on osteoblasts/osteocytes with novel analogs. Bone. 2006;39(3): 443-452.

10. Fleisch H. Bisphosphonates: mechanisms of action. Endocr Rev. 1998; 19(1):80-100.

11. Mashiba T, Mori S, Burr DB, et al. The effects of suppressed bone remodeling by bisphosphonates on microdamage accumulation and degree of mineralization in the cortical bone of dog rib. J Bone Miner Metab. 2005;23(Suppl):36-42.

12. Diniz-Freitas M, Limeres J. Prevention of medication-related osteonecrosis of the jaws secondary to tooth extractions. A systematic review. Med Oral Patol Oral Cir Bucal. 2016;21(2):e250-e259.

13. Mkele G. The safety and efficacy of osteoporosis therapy: review. SA Pharm Jl. 2015;82(9):29-32.

14. Bain S, Jerome C, Shen V, Dupin-Roger I, Ammann P. Strontium ranelate improves bone strength in ovariectomized rat by positively influencing bone resistance determinants. Osteoporos Int. 2009;20(8): $1417-1428$.

15. Arlot ME, Jiang Y, Genant HK, et al. Histomorphometric and microCT analysis of bone biopsies from postmenopausal osteoporotic women treated with strontium ranelate. J Bone Miner Res. 2008;23(2): 215-222.

16. Lima H, Maia J, Bandeira F. Trajectories of bone remodeling markers and bone mineral density during treatment with strontium ranelate in postmenopausal women previously treated with bisphosphonates. Clin Med Insights Endocrinol Diabetes. 2014;7:7-11.

17. Lin JH, Russell G, Gertz B. Pharmacokinetics of alendronate: an overview. Int J Clin Pract Suppl. 1999;101:18-26.

18. Papapoulos SE, Cremers SC. Prolonged bisphosphonate release after treatment in children. $N$ Engl J Med. 2007;356(10):1075-1076.

19. Drake MT, Clarke BL, Khosla S. Bisphosphonates: mechanism of action and role in clinical practice. Mayo Clin Proc. 2008;83(9):1032-1045.

20. Khosla S, Burr D, Cauley J, et al. Bisphosphonate-associated osteonecrosis of the jaw: report of a task force of the American Society for Bone and Mineral Research. J Bone Miner Res. 2007;22(10):1479-1491. 
21. Narongroeknawin P, Danila MI, Humphreys LG Jr, Barasch A, Curtis JR. Bisphosphonate-associated osteonecrosis of the jaw, with healing after teriparatide: a review of the literature and a case report. Spec Care Dentist. 2010;30(2):77-82.

22. Marx RE, Cillo JE Jr, Ulloa JJ. Oral bisphosphonate-induced osteonecrosis: risk factors, prediction of risk using serum CTX testing, prevention, and treatment. J Oral Maxillofac Surg. 2007;65(12): 2397-2410.

23. Kwon YD, Kim DY, Ohe JY, Yoo JY, Walter C. Correlation between serum C-terminal cross-linking telopeptide of type I collagen and staging of oral bisphosphonate-related osteonecrosis of the jaws. J Oral Maxillofac Surg. 2009;67(12):2644-2648.
24. Lazarovici TS, Mesilaty-Gross S, Vered I, et al. Serologic bone markers for predicting development of osteonecrosis of the jaw in patients receiving bisphosphonates. J Oral Maxillofac Surg. 2010;68(9): 2241-2247.

25. Payne JB, Stoner JA, Lee HM, Nummikoski PV, Reinhardt RA, Golub LM. Serum bone biomarkers and oral/systemic bone loss in humans. J Dent Res. 2011;90(6):747-751.

26. Enciso R, Keaton J, Saleh N, Ahmadieh A, Clark GT, Sedghizadeh PP. Assessing the utility of serum C-telopeptide cross-link of type 1 collagen as a predictor of bisphosphonate-related osteonecrosis of the jaw: A systematic review and meta-analysis. J Am Dent Assoc. 2016;147(7): $551-560$ e511.
Clinical Interventions in Aging

\section{Publish your work in this journal}

Clinical Interventions in Aging is an international, peer-reviewed journal focusing on evidence-based reports on the value or lack thereof of treatments intended to prevent or delay the onset of maladaptive correlates of aging in human beings. This journal is indexed on PubMed Central, MedLine,

\section{Dovepress}

CAS, Scopus and the Elsevier Bibliographic databases. The manuscript management system is completely online and includes a very quick and fair peer-review system, which is all easy to use. Visit http://www.dovepress. com/testimonials.php to read real quotes from published authors. 